\title{
Revealing the Mass Loss Structures of Four Key Massive Binaries Using Optical Spectropolarimetry
}

\author{
Jamie R. Lomax \\ Homer L. Dodge Department of Physics and Astronomy, University of Oklahoma, Norman, \\ OK, 73019, USA \\ email: Jamie.R.Lomax@ou.edu
}

\begin{abstract}
The majority of massive stars are members of binary systems. However, in order to understand their evolutionary pathways, mass and angular momentum loss from these systems needs to be well characterized. Self-consistent explanations for their behavior across many wavelength regimes need to be valid in order to illuminate key evolutionary phases. I present the results of linear spectropolarimetric studies of three key binaries ( $\beta$ Lyrae, V356 Sgr, V444 Cyg, and WR 140) which reveal important geometric information about their circumstellar material. $\beta$ Lyrae exhibits a repeatable discrepancy between secondary eclipse in the total and polarized light curves that indicates an accretion hot spot has formed on the edge of the disk in the system. The existence of this hot spot and its relationship to bipolar outflows within the system is important in the understanding of mass transfer dynamics in Roche-lobe overflow binaries. Preliminary work on V356 Sgr suggests the system maybe surrounded by a common envelope. V444 Cyg shows evidence that its shock creates a cone with a large opening angle of missing material around the WN star. This suggests the effects of radiative inhibition or braking, can be significant contributors to the location and shape of the shock within colliding wind binaries. The intrinsic polarization component of WR 140 is likely due to the formation of dust within the system near periastron passages. Continued work on these and additional objects will provide new and important constraints on the mass loss structures within binary systems.
\end{abstract}

Keywords. techniques: photometric, (stars:) binaries: eclipsing, stars: individual (beta Lyr, V356 Sgr, V444 Cyg, WR 140)

\section{Introduction}

Podsiadlowski et al. (1992) showed that between 30 and $50 \%$ of massive binaries have initial periods that are short enough (under 1500 days) for the individual stars to interact within a system. Additional work by Sana et al. (2012) suggests that up to $70 \%$ of all massive stars are members of a binary at some point during their lives. Given the importance of mass loss on massive star evolution, it is imperative that we better understand how binary systems exchange and lose material.

Polarization studies of massive binaries can give important, geometric information about the mass loss structures that form from strong stellar winds and during phases of Roche lobe overflow. This is particularly useful for understanding mass loss in systems which remain unresolved. Below I describe the results of polarimetric studies of four massive binary systems: two Roche lobe overflow objects and two colliding-wind binaries. 

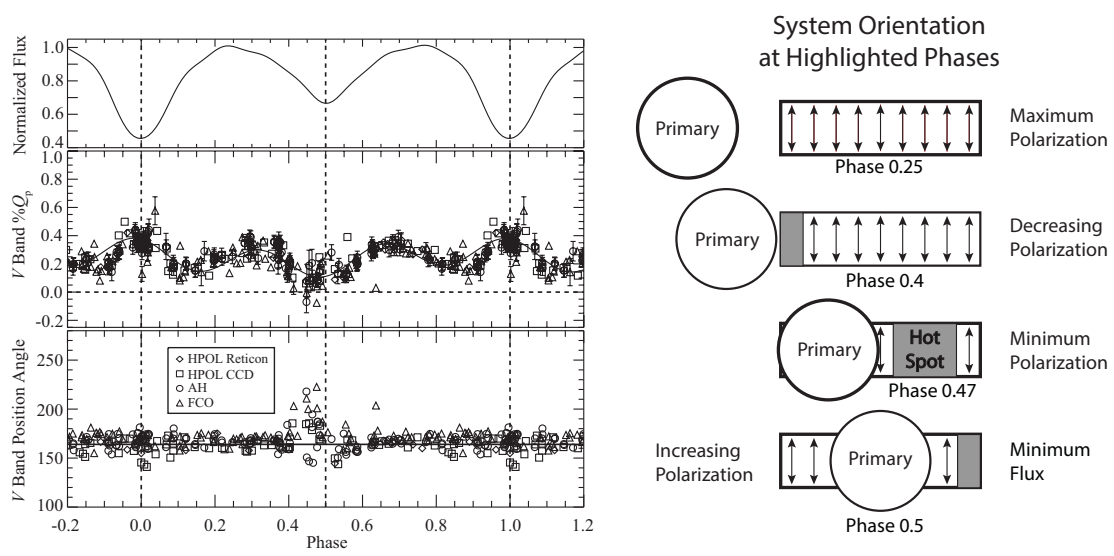

Figure 1. $V$ Band Polarization of $\beta$ Lyrae (Lomax et al. 2012). The top left panel is the normalized $V$ band light curve from Harmanec et al. (1996). The middle and bottom left panels display the intrinsic polarization and position angle versus phase. Dashed vertical lines indicated eclipse locations. The solid curve in the middle panel represents the Fourier fit to the data. The solid horizontal line in the bottom panel indicates the error-weighted mean position angle of the system. Point styles indicate the source of the data. The right panel shows the system orientation including the location of the hot spot at different phases as indicated.

\section{Roche-Lobe Overflow}

\section{1. $\beta$ Lyr}

$\beta$ Lyrae is a semi-detached, eclipsing binary. The primary, B6-8 II, giant star is losing material via Roche lobe overflow to the secondary B0.5 main sequence star (Hubeny \& Plavec 1991). A thick accretion disk has formed around the secondary which almost completely obscures its interior. Bipolar outflows are also known to exist in the system (Harmanec et al. 1996; Hoffman et al. 1998).

Figure 1 shows the polarization behavior of $\beta$ Lyrae with phase (Lomax et al. 2012). The average position angle of the polarization, excluding the behavior around secondary eclipse, is consistent with the orientation of the disk and system on the sky, indicating that the broad band polarization is caused by scattering in the disk. Three distinct features appear in the $\% Q_{p}$. The first is the increase in polarization at primary eclipse caused by a decrease in the unpolarized flux due to the disk blocking the star losing material. Second, there are local maxima at quadurature phases due to light scattering off the edge of the disk. The last feature is a secondary eclipse that occurs at phase $0.481 \pm 0.001$ that is associated with a position angle rotation. This is consistent with a hot spot forming on the edge of the accretion disk due to the impact of the mass stream (Lomax et al. 2012) and has been confirmed through modeling of $\beta$ Lyr's light curve (Mennickent \& Djurašević 2013). Size estimates derived from the polarimetric data indicated that the hot spot has a width of approximately $22-33 R_{\odot}$ across the disk edge (Lomax et al. 2012 , the disk diameter is $60 R_{\odot}$ for comparison).

\section{2. $V 356 \mathrm{Sgr}$}

There are two major differences between the $\beta$ Lyr system and V356 Sgr that should be kept in mind when comparing the two systems. The first is the disk in $\beta$ Lyr is eclipsed at phase 0.5 while it is eclipses at phase 0.0 in V356 Sgr. This is because V356 Sgr has an A2 supergiant that is losing mass to its brighter, primary B star (Wilson \& Caldwell 1978). Additionally, the accretion disk that has formed around the primary star is optically thin (Wilson \& Caldwell 1978), where as $\beta$ Lyr's is optically thick. 


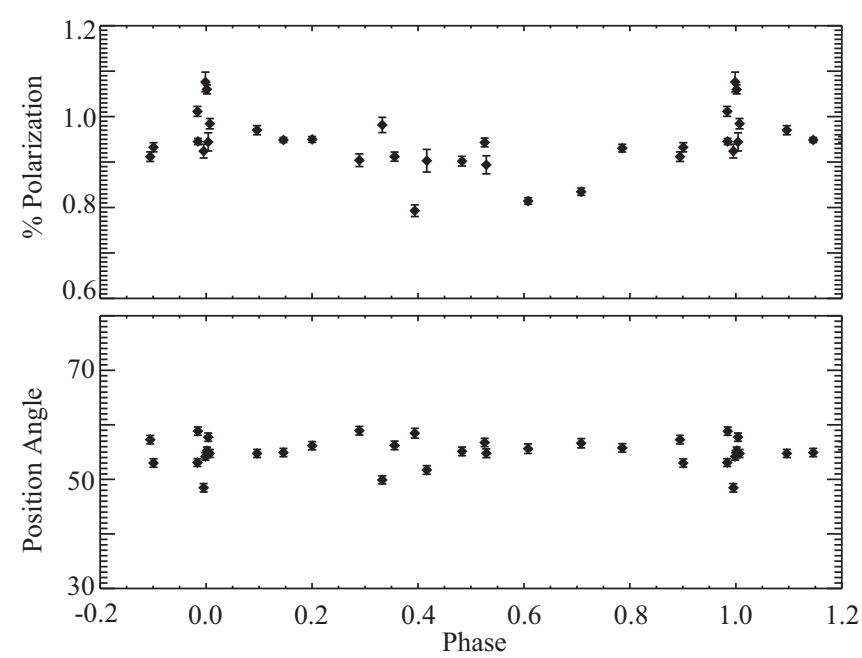

Figure 2. $V$ Band Polarization of V356 Sgr (Malatesta et al. 2013 AAS proceedings). The top panel displays the total polarization (interstellar polarization has not been removed) versus phase while the bottom panel shows the position angle versus phase.

Figure 2 displays preliminary polarization curves of V356 Sgr (Malatesta et al. 2013 AAS proceedings). The relative lack of eclipse effects compared to $\beta$ Lyr and the lack of polarization features at quadrature phases, which if they did exist would be due to scattering off the disk edge, indicate that the scattering region in V356 Sgr might be circumbinary material, such as a common envelope, or a combination of circumbinary material and the disk within the system.

\section{Colliding Wind Binaries}

\subsection{V444 Cyg}

V444 Cyg is an eclipsing WR+O system with a short 4.2 day period. Evidence for colliding winds exists in a variety data from IUE, ASCA, ROSAT, Einstein, and XMMNewton despite a naive estimate (based on mass-loss rates and terminal velocities) that the wind of the WR star should impact the surface of the O star instead of forming a shock (Moffat et al. 1982; Koenigsberger \& Auer 1985; Pollock 1987; Shore \& Brown 1988; Corcoran et al. 1996; Maeda et al. 1999; Fauchez et al. 2011). Theoretical work has suggested that the situation is more complex; radiative braking and radiative inhibition may play an important roll in the location of the shock (Owocki \& Gayley 1995; Stevens \& Pollock 1994).

Figure 3 shows the polarization behavior of V444 Cyg's HeII 4686 with phase. The Stokes $\% U_{p}$ parameter (the $p$ indicates that the standard Stokes parameters have been rotated so that the error-weighted mean $\% Q$ is zero) has a different polarization behavior around secondary eclipse compared to other phases, where as the Stokes $\% Q_{p}$ parameter appears to scatter about $0 \%$ more equally. To show this I have calculated the error weighted mean for each parameter in the 0.3 to 0.75 , and the 0.75 to 1.3 phase regions. Figure 3 shows that the error weighted mean $\% U_{p}$ is more negative around secondary eclipse than at other phases at a $2 \sigma$ significance. This type of behavior is very similar to the behavior of modeled polarization for a single degenerate Type Ia supernovae where the companion carves a cavity out of the ejecta (Kasen et al. 2004). In that scenario viewing angles close to the cavity produce a more negative polarization than viewing 


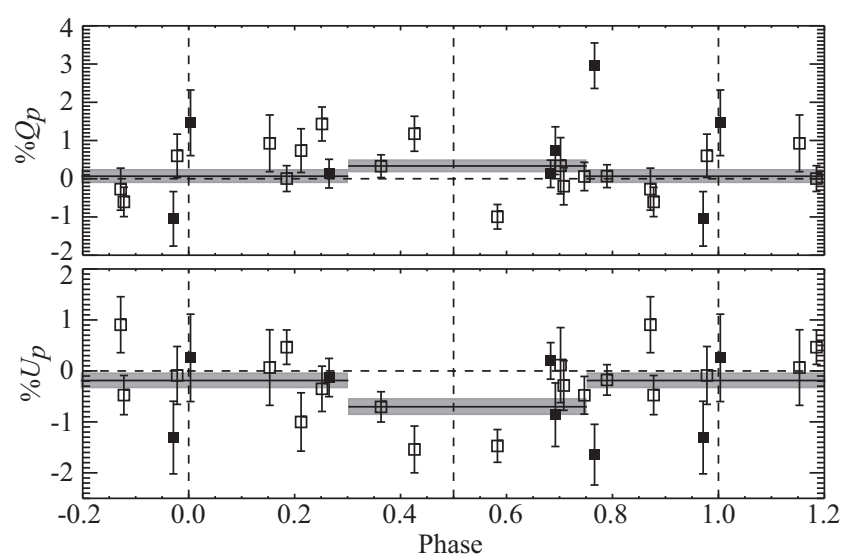

Figure 3. HeII 4686 Polarization of V444 Cyg. The top panel displays the Stokes $Q$ parameter and the bottom panel displays the Stokes $U$ parameter. Solid filled squares indicate data taken with HPOL at Ritter Observatory while open symbols are HPOL data from the Pine Bluff Observatory. Horizontal dashed lines indicate zero points for each parameter, while vertical dashed lines make eclipse phases. Grey boxes represent the $1 \sigma$ uncertainty on the error-weighted mean (solid line inside the box) Stokes parameter for the phase range over which it is plotted.

angles farther away. For V444 Cyg, the cavity is carved out of the WN-star wind by the shock and O-star wind while viewing angles close to the cavity occur around secondary eclipse.

\subsection{WR 140}

WR 140 is the canonical long period, high eccentricity WR+O binary system. Periastron passage sparks the production of dust, which quickly expands, dissipates, and is destroyed (Monnier et al. 2002; Taranova \& Shenavrin 2011). Previous studies of WR 140 have indicated that the wavelength dependent polarization signatures observed from the system are consistent with dust. Figure 4 shows the polarization light curve of the system. The points show large variability after the 1985 periastron passage that does not exist before the 1993 periastron passage. This is also consistent with dust being the primary scattering region in the system.

\section{Future Work}

The results of these studies show that polarization is a powerful tool for revealing the mass loss structures around binary systems. Despite $\beta$ Lyr and V356 Sgr being very similar systems, their polarization light curves are very different. This suggests that a large scale polarimetric survey of Roche lobe overflow systems is needed to better characterize their circumstellar and circumbinary material. The difference in mean polarization of V444 Cyg at secondary eclipse compared to other phases is currently only suggestive at a $2 \sigma$ detection level. Work is ongoing to obtain more observations to fill in the light curve and better characterize this behavior. Polarimetric monitoring of WR 140 has historically been poor. Large gaps between epochs of data exist at crucial dust production phases. Better observational cadence, particularly through periastron passage, could potentially reveal new information about the formation and destruction of dust in the system.

\section{Acknowledgements}

This research includes contributions from the following collaborators: Jennifer L 


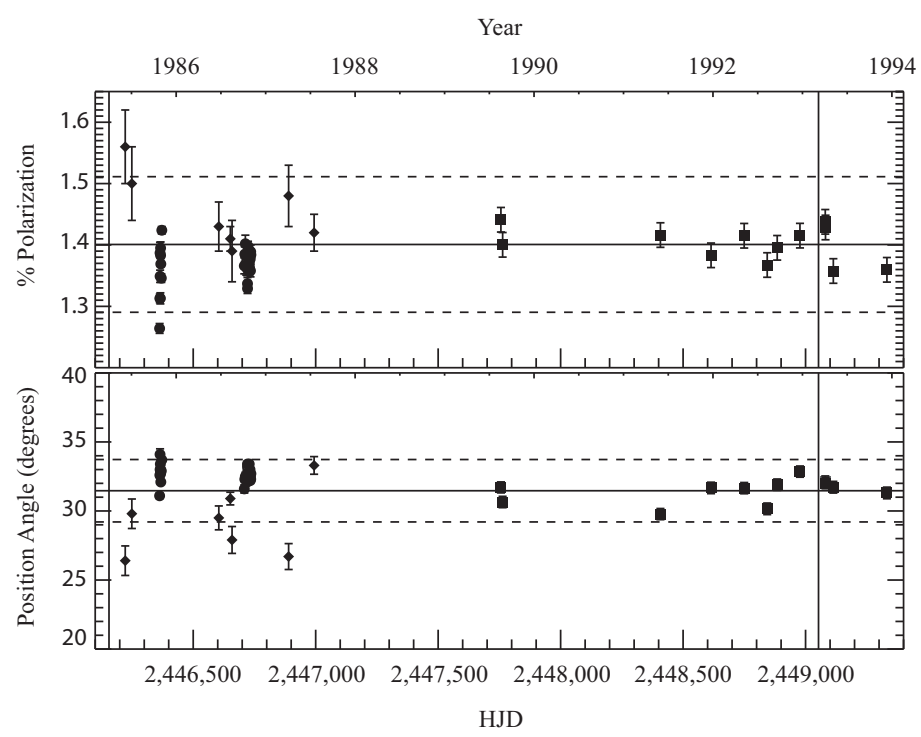

Figure 4. Polarization of WR 140. Diamonds indicate data taken with the Lyot instrument at Pine Bluff Observatory, squares are data from HPOL at the Pine Bluff Observatory, and circles indicate archival data from Robert et al. (1989).

Hoffman (The University of Denver), James Davidson (University of Toledo), John Wisniewski (University of Oklahoma), and Michael Malatesta (University of Oklahoma).

\section{References}

Corcoran, M. F., Stevens, I. R., Pollock, A. M. T., et al. 1996, ApJ 464, 434

Fauchez, T., De Becker, M., \& Nazé, Y. 2011, Bulletin de la Societe Royale des Sciences de Liege 80, 673

Harmanec, P., Morand, F., Bonneau, D., et al. 1996, A\&A 312, 879

Hoffman, J. L., Nordsieck, K. H., \& Fox, G. K. 1998, AJ 115, 1576

Hubeny, I. \& Plavec, M. J. 1991, AJ 102, 1156

Kasen, D., Nugent, P., Thomas, R. C., \& Wang, L. 2004, ApJ 610, 876

Koenigsberger, G. \& Auer, L. H. 1985, ApJ 297, 255

Lomax, J. R., Hoffman, J. L., Elias, II, N. M., Bastien, F. A., \& Holenstein, B. D. 2012, ApJ 750,59

Maeda, Y., Koyama, K., Yokogawa, J., \& Skinner, S. 1999, ApJ 510, 967

Mennickent, R. E. \& Djurašević, G. 2013, MNRAS 432, 799

Moffat, A. F. J., Firmani, C., McLean, I. S., \& Seggewiss, W. 1982, in C. W. H. De Loore \& A. J. Willis (eds.), Wolf-Rayet Stars: Observations, Physics, Evolution, Vol. 99 of IAU Symposium, pp 577-581

Monnier, J. D., Tuthill, P. G., \& Danchi, W. C. 2002, ApJ (Letters) 567, L137

Owocki, S. P. \& Gayley, K. G. 1995, ApJ (Letters) 454, L145

Podsiadlowski, P., Joss, P. C., \& Hsu, J. J. L. 1992, ApJ 391, 246

Pollock, A. M. T. 1987, ApJ 320, 283

Robert, C., Moffat, A. F. J., Bastien, P., \& Drissen, L., St.-Louis, N. 1989, ApJ 347, 1034

Sana, H., de Mink, S. E., de Koter, A., et al. 2012, Science 337, 444

Shore, S. N. \& Brown, D. N. 1988, ApJ 334, 1021

Stevens, I. R. \& Pollock, A. M. T. 1994, MNRAS 269, 226

Taranova, O. G. \& Shenavrin, V. I. 2011, Astronomy Letters 37, 30

Wilson, R. E. \& Caldwell, C. N. 1978, ApJ 221, 917 


\section{Discussion}

PeTERs: Eclipse of V356 Sgr in the FUSE FUV definitely shows the presence of a jet structure that emits strongly in Ovi (implies a 300,000 K plasma). Could this structure produce a polarization signature?

Lomax: Yes. In $\beta$ Lyr we see a polarization signature from the jets in the UV continuum and the emission lines in the optical. However, we don't see similar signatures in V356 Sgr.

DAVID-URAZ: On the WR 140 polarization curve there doesn't seem to be as much variability after the 1993 periastron; is that just lack of data?

LOMAX: I do think we've missed a lot of variability because of lack of observations, particularly in that periastron passage, but maybe even at other times as well.

DAVID-URAZ: Were there any polarization observations during the 2010 periastron campaign?

Lomax: No. There is an unpublished observation from 2013, but nothing that occurred during the 2010 periastron passage.

WADE: What are the systematic contributions to scatter in $\beta$ Lyr or V356 Sgr? In other words, how close to the photon limit are your uncertainties?

Lomax: Systematic uncertainties for the HPOL data are small and about $\pm 0.02 \%$ in the broadbands. A paper currently in prep. (Davidson et al.) quotes this in a more complete manner with more exact numbers and a comparison to how our systematic uncertainties change with time. I am uncertain about the other data because it was all archival and I haven't been able to find information for those instruments.

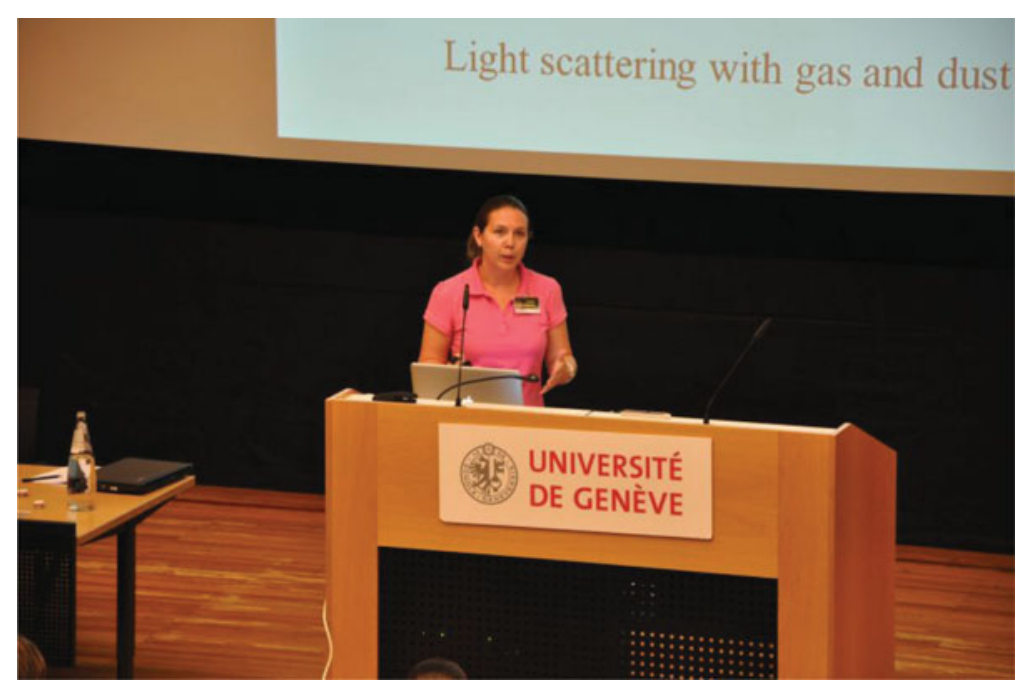

Jamie Lomax 